\title{
OTIMIZAÇÃO DAS CONDIÇÕES EXPERIMENTAIS DA HIDRÓLISE DA SACAROSE
}

\author{
C.P.B. QUITETE ${ }^{1}$, V.L. XIMENES ${ }^{1}$, A.C. FRAGA $^{1}$ e B.G. PALMA ${ }^{1}$ \\ ${ }^{1}$ PETROBRAS / CENPES \\ E-mail para contato: crispontes@ petrobras.com.br
}

\begin{abstract}
RESUMO - Neste estudo, a reação de hidrólise da sacarose foi realizada empregando como catalisador a resina sulfônica comercial Amberlyst 36, obtendo conversão próxima a $100 \%$ e seletividades à glicose e frutose próximas a 50\% após otimização. Um planejamento fatorial $2^{2}$ com três réplicas no ponto central foi proposto para avaliar o efeito das variáveis temperatura e tempo reacional na conversão da reação. Como reagente, foi utilizada uma solução aquosa de sacarose com concentração de $60 \% \mathrm{~m} / \mathrm{m}$. A quantidade de catalisador foi definida a partir da razão mássica entre catalisador e sacarose, para um valor de $4,2 \% \mathrm{~m} / \mathrm{m}$. Os níveis dos fatores foram $35^{\circ} \mathrm{C}, 55^{\circ} \mathrm{C}$ e $75^{\circ} \mathrm{C}$, para a temperatura, e $0,5 \mathrm{~h}, 2,0 \mathrm{~h}$ e $3,5 \mathrm{~h}$, para o tempo de reação. Em modo contínuo, o desempenho da Amberlyst 36 na reação de hidrólise da sacarose foi investigado em reator de leito fixo, indicando que a desativação foi bem pequena nas condições da reação, durante o período de 48 horas. Os parâmetros cinéticos foram determinados nas três temperaturas, usando oito tempos de amostragem.
\end{abstract}

\section{INTRODUÇÃO}

A sacarose é o principal componente do caldo da cana-de-açúcar. Sua molécula contém dois monossacarídeos, glicose e frutose, unidos por uma ligação glicosídica do tipo $\alpha-1, \beta-2$. A quebra desta ligação através de sua hidrólise fornece glicose e frutose em proporção equimolar. Outros dissacarídeos, como a celobiose (ligação $\beta-1,4$ ) e a maltose (ligação $\alpha-1,4$ ), são usados como compostos modelos para estudar a hidrólise da celulose e do amido. As ligações glicosídicas $\alpha-1,4$ e $\beta-1,4$ são muito menos reativas que a ligação $\alpha-1, \beta-2$, tornando a sacarose um dissacarídeo mais suscetível à hidrólise (Marzo et al., 2012; Quitete et al., 2013). A lise da ligação glicosídica pode ser realizada com enzimas ou ácidos inorgânicos, como ácido sulfúrico e ácido clorídrico. Os ácidos inorgânicos, apesar de serem muito eficientes, possuem desvantagens como o elevado potencial de corrosividade, a maior degradação dos açúcares e a necessidade de tratamento de rejeitos. Uma alternativa à catálise homogênea é o uso de sólidos ácidos, como as resinas de troca iônica (ex.: Amberlyst 36, Amberlite IR 200), as zeólitas, entre outros (Marzo et al., 2012; Abbadi et al., 1998; Bodamer e Kunin, 1951).

A resina Amberlyst 36 contém grupos sulfônicos em sua estrutura, possuindo uma elevada acidez de Bronsted $\left(4,7 \mathrm{mmol} \mathrm{H}^{+} / \mathrm{g}\right)$, o que lhe confere atividade comprovada na reação de hidrólise da sacarose (Bodamer e Kunim, 1951). Além disso, a Amberlyst 36 possui um tamanho médio de poros de $24 \mathrm{~nm}$, valor alto em relação ao tamanho da molécula de sacarose (1 a $2 \mathrm{~nm}$ ), o que minimiza o efeito difusional intrapartícula. Outra vantagem deste catalisador é o elevado 


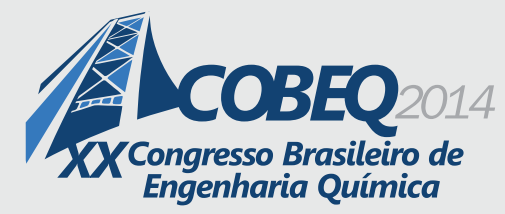

19 a 22 de outubro de 2014

Florianópolis/SC

grau de reticulação da resina, o que torna seus sítios ácidos ainda mais acessíveis (Whyman, 2013).

O principal objetivo deste trabalho foi avaliar o desempenho de uma resina de troca iônica com sítios sulfônicos na reação de hidrólise de uma corrente sintética de xarope de cana, empregando para isso a resina comercial Amberlyst 36 e uma solução concentrada de sacarose. $\mathrm{O}$ produto da hidrólise do xarope pode ser empregado em outros processos, como, por exemplo, na hidrogenação de glicose e frutose a sorbitol e manitol respectivamente, que podem ser convertidos posteriormente a isossorbida e isomanida (Almeida et al., 2010).

\section{MATERIAIS E MÉTODOS}

As reações de hidrólise foram conduzidas em um reator batelada com agitação magnética, acoplado a um condensador de refluxo e com controle de temperatura através de um banho termostático. A concentração de sacarose (fornecida pela Sigma-Aldrich) empregada foi $60 \% \mathrm{~m} / \mathrm{m}$ e a razão catalisador/sacarose igual a $4,2 \% \mathrm{~m} / \mathrm{m}$. Um planejamento fatorial $2^{2}$ com três réplicas no ponto central foi utilizado para avaliação do efeito da temperatura e do tempo de reação na hidrólise da sacarose com a resina Amberlyst 36. Os níveis para a temperatura foram $35^{\circ} \mathrm{C}, 55^{\circ} \mathrm{C}$ e $75^{\circ} \mathrm{C}$, nos tempos de $0,5 \mathrm{~h}, 2,0 \mathrm{~h}$ e $3,5 \mathrm{~h}$. Curvas cinéticas foram obtidas nas três temperaturas para determinação da energia de ativação. As análises de todos os produtos foram realizadas em um HPLC da Agilent Technologies série 1100, com a coluna AMINEX HPX 87 H, capaz de detectar carboidratos, ácidos orgânicos e compostos de degradação (ex.: 5-HMF e furfural). A estabilidade térmica dos açúcares na forma anidra (glicose, frutose e sacarose) foi determinada através de análise termogravimétrica em atmosfera inerte, usando uma vazão de nitrogênio de $40 \mathrm{ml} / \mathrm{min}$ e uma taxa de aquecimento de $10^{\circ} \mathrm{C} / \mathrm{min}$, até $600^{\circ} \mathrm{C}$. Após os testes em batelada, a hidrólise foi conduzida em unidade contínua, utilizando um reator de leito fixo operando em fluxo ascendente, e sob as condições de $75^{\circ} \mathrm{C}$ e LHSV de $2 \mathrm{~h}^{-1}$.

\section{RESULTADOS E DISCUSSÃO}

\subsection{Determinação dos parâmetros cinéticos}

A ordem da reação foi determinada através do método integral para as três temperaturas, confirmando os resultados da literatura, que indicavam que a reação de hidrólise da sacarose segue uma cinética de primeira ordem (Buttersack e Laketic, 1994). Conhecendo o modelo cinético que rege a reação, a velocidade específica de reação foi determinada para as três temperaturas, permitindo a determinação da energia de ativação da reação ao utilizar a Amberlyst 36 como catalisador, através da equação de Arrhenius. Os valores encontrados para as velocidades específicas foram $0,00074 \mathrm{~mol} / \mathrm{min}^{-1}$ para $35^{\circ} \mathrm{C}, 0,00302 \mathrm{~mol} / \mathrm{min}^{-1}$ para $55^{\circ} \mathrm{C}$ e $0,01462 \mathrm{~mol} / \mathrm{min}^{-1}$ para $75^{\circ} \mathrm{C}$, fornecendo uma energia de ativação de $66,3 \mathrm{~kJ} / \mathrm{mol}$. As curvas 
para as três temperaturas podem ser vistas na Figura 1.

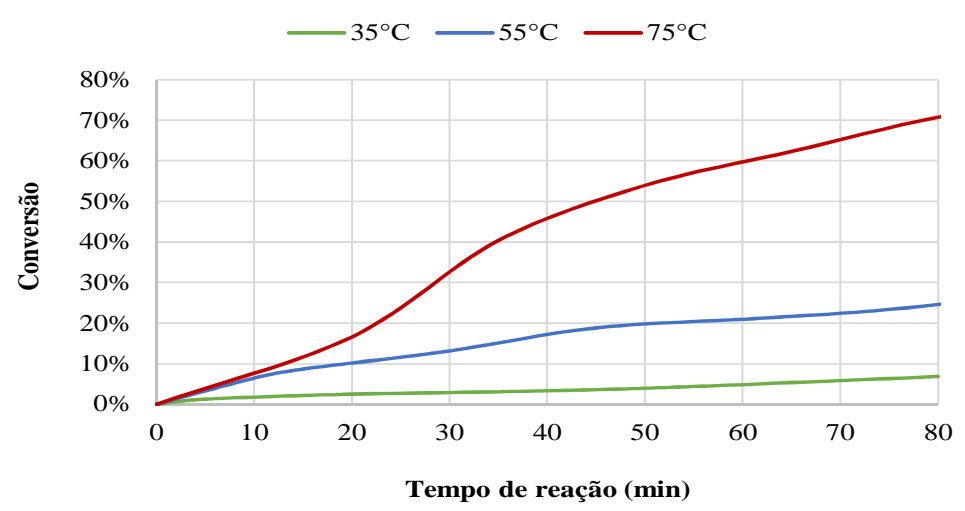

Figura 1 - Curvas cinéticas para a hidrólise da sacarose em três diferentes temperaturas.

A formação de 5-HMF no meio reacional aumenta com o tempo, como mostra a Figura 2, alcançando um valor de $488 \mathrm{mg} / \mathrm{kg}$ após 140 minutos de reação, estando em acordo com os dados publicados por Moreau et al. (2000). A produção de 5-HMF está relacionada à formação de ácidos húmicos e resinas (Carlini et al., 2010), o que promove a coloração do hidrolisado. Essa questão é fundamentalmente importante na indústria de alimentos, mas também possui relevância caso o produto da hidrólise seja usado como carga de outro processo, pois a presença de subprodutos pode vir a causar desativação dos catalisadores das etapas à jusante.

Além do tempo, a temperatura e a concentração de catalisador possuem efeito na formação de 5-HMF. Moreau et al. (2000) encontraram maiores concentrações de 5-HMF ao aumentar a temperatura, alcançando valores iguais a $17 \mathrm{mg} / \mathrm{kg}, 685 \mathrm{mg} / \mathrm{kg}$ e $825 \mathrm{mg} / \mathrm{kg}$ a $75^{\circ} \mathrm{C}, 85^{\circ} \mathrm{C}$ e $95^{\circ} \mathrm{C}$, respectivamente. A conversão de sacarose foi igual a $72,5 \%$ e $100 \%$ nas demais temperaturas. Logo, os níveis de temperatura $\left(35^{\circ} \mathrm{C}\right.$ e $\left.75^{\circ} \mathrm{C}\right)$ empregados no planejamento foram escolhidos através destas informações. 


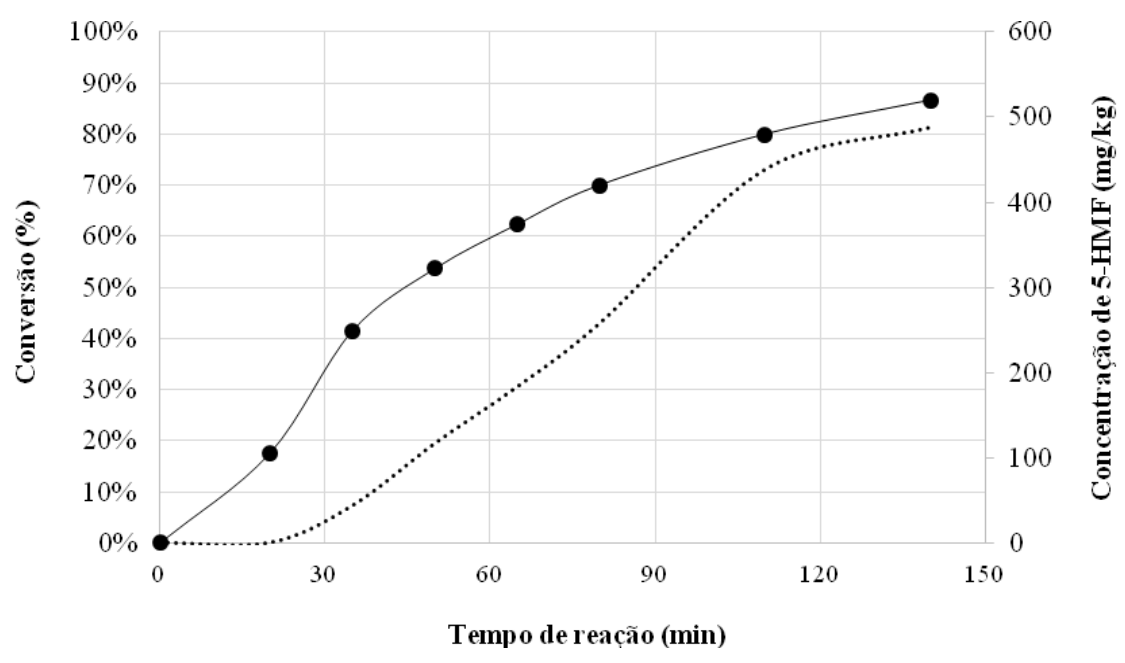

Figura 2 - Curvas cinéticas obtidas a partir a $75^{\circ} \mathrm{C}$ e a concentração de 5-HMF formada durante a reação.

\subsection{Planejamento experimental e avaliação estatística}

Os resultados de conversão da sacarose para o planejamento proposto, que seguiu uma distribuição normal e foram analisados pelo teste t-Student, são apresentados na Tabela 1. Observou-se que a seletividade à glicose e à frutose foram próximas a $50 \%$ nas condições estudadas. Somente a temperatura e o tempo reacional foram importantes a um nível de $5 \%$ de significância. A interação dos fatores não apresentou efeito relevante, não sendo apresentada na Figura 3.

Tabela 1 - Condições experimentais e resultados de conversão do planejamento fatorial.

\begin{tabular}{ccc} 
Temperatura & Tempo & Conversão \\
\hline $35^{\circ} \mathrm{C}$ & $0,5 \mathrm{~h}$ & $3 \%$ \\
$35^{\circ} \mathrm{C}$ & $3,5 \mathrm{~h}$ & $13 \%$ \\
$55^{\circ} \mathrm{C}$ & $2,0 \mathrm{~h}$ & $39 \%$ \\
$55^{\circ} \mathrm{C}$ & $2,0 \mathrm{~h}$ & $40 \%$ \\
$55^{\circ} \mathrm{C}$ & $2,0 \mathrm{~h}$ & $45 \%$ \\
$75^{\circ} \mathrm{C}$ & $0,5 \mathrm{~h}$ & $63 \%$ \\
$75^{\circ} \mathrm{C}$ & $3,5 \mathrm{~h}$ & $97 \%$
\end{tabular}




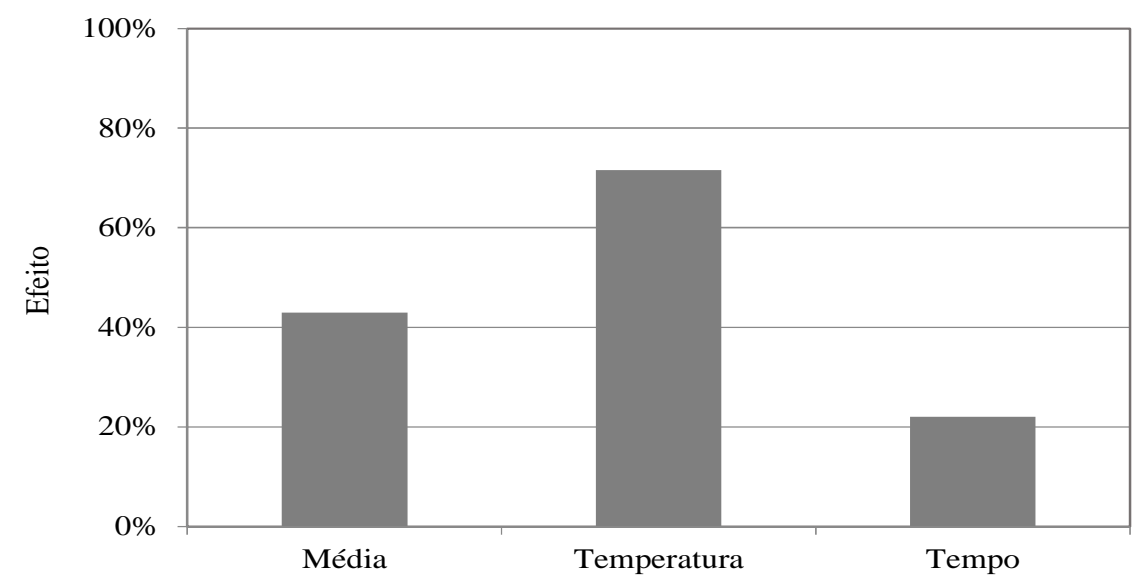

Figura 3 - Gráfico de Pareto para o planejamento experimental.

O coeficiente de determinação $\left(\mathrm{R}^{2}\right)$ foi igual a $99,4 \%$, indicando que $99,4 \%$ da variação total poderia ser explicada pela regressão e $0,6 \%$ pelos resíduos. O modelo obtido para a conversão de sacarose (Y) e a análise dos coeficientes da regressão são apresentados na Equação 2 e na Tabela 2:

$Y=42,96+35,78 \times T+11,00 \times t$

(Equação 2)

Tabela 2 - Parâmetros do modelo obtido através do planejamento experimental.

\begin{tabular}{l|cccc}
\multicolumn{1}{r|}{ Fatores } & Coeficiente (\%) & $\begin{array}{c}\text { Erro padrão do } \\
\text { coeficiente }(\%)\end{array}$ & IC (-95\%) & IC (+95\%) \\
\hline Média & 42,956 & 1,08 & 38,33 & 47,58 \\
Temperatura (L) & 35,781 & 1,42 & 29,66 & 41,90 \\
Tempo (L) & 11,002 & 1,42 & 4,88 & 17,12
\end{tabular}

A Figura 4 mostra que é possível obter uma elevada conversão da sacarose a $75^{\circ} \mathrm{C}$, já em um tempo de reação inferior a uma hora. Entretanto, em níveis inferiores de temperatura, as conversões obtidas não atingem o patamar desejado, mesmo em elevados tempos de reação. Deve-se avaliar a influência da temperatura na formação de produtos de degradação ao empregar 
as resinas.

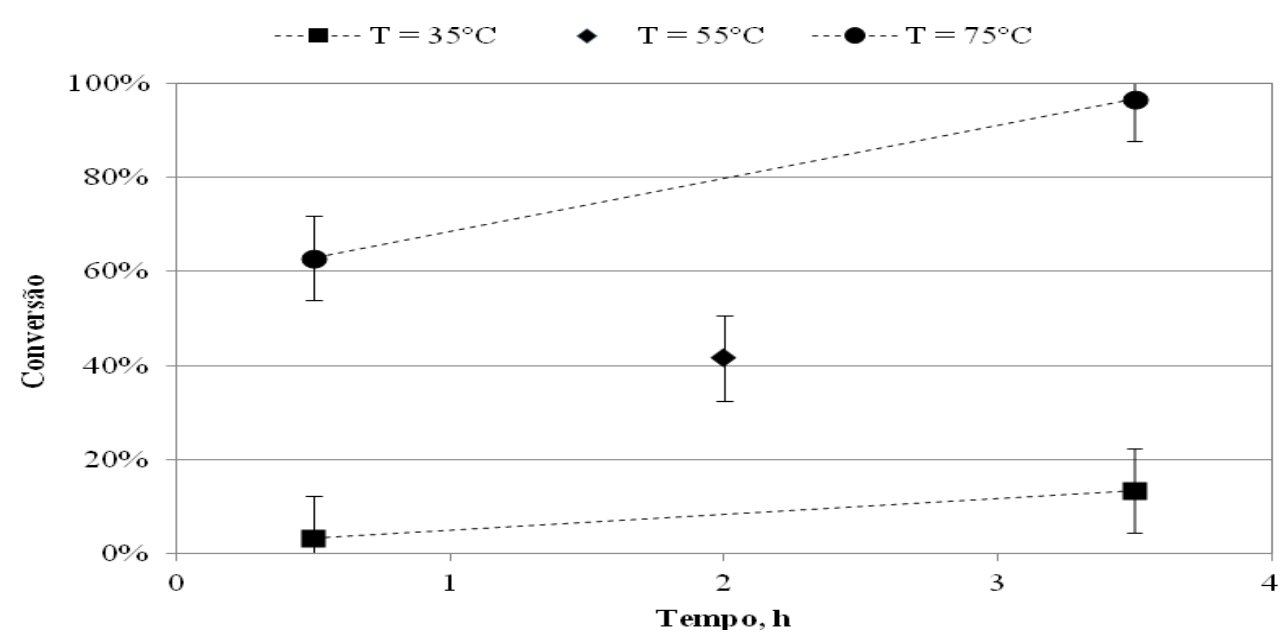

Figura 4 - Resultados do planejamento experimental nas condições testadas.

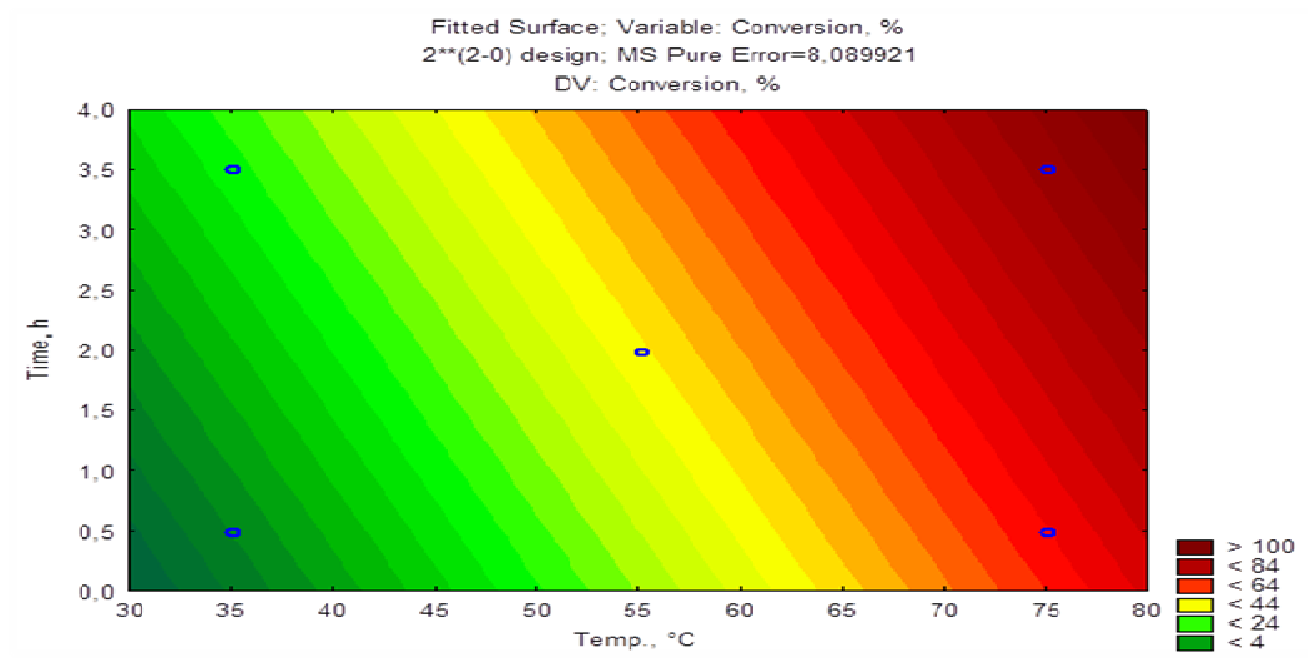

Figura 5 - Curvas de níveis para o modelo obtido através do planejamento experimental.

\subsection{Avaliação da reação em regime contínuo}

Durante o teste conduzido utilizando um leito fixo em reator PFR, o catalisador apresentou pequeno decréscimo na conversão. Após $48 \mathrm{~h}$ de reação, a conversão caiu de $98 \%$ para $95 \%$, indicando uma redução de atividade de aproximadamente 3\%, conforme apresentado na Figura 6. 
A seletividade à glicose permaneceu ligeiramente maior do que para a frutose, com valores de $55 \%$ e $45 \%$, respectivamente. Teoricamente, esperava-se uma relação molar de 1:1 entre os dois monossacarídeos, de acordo com a estequiometria da reação. Uma provável explicação é a ocorrência de reações paralelas a partir da frutose, já que esta possui menor estabilidade que a glicose e a sacarose, fato verificado através da análise térmica. Na Tabela 3 são apresentados os eventos relevantes durante a análise termogravimétrica realizada nos açúcares, apontando as temperaturas relativas aos processo de fusão e de caramelização.

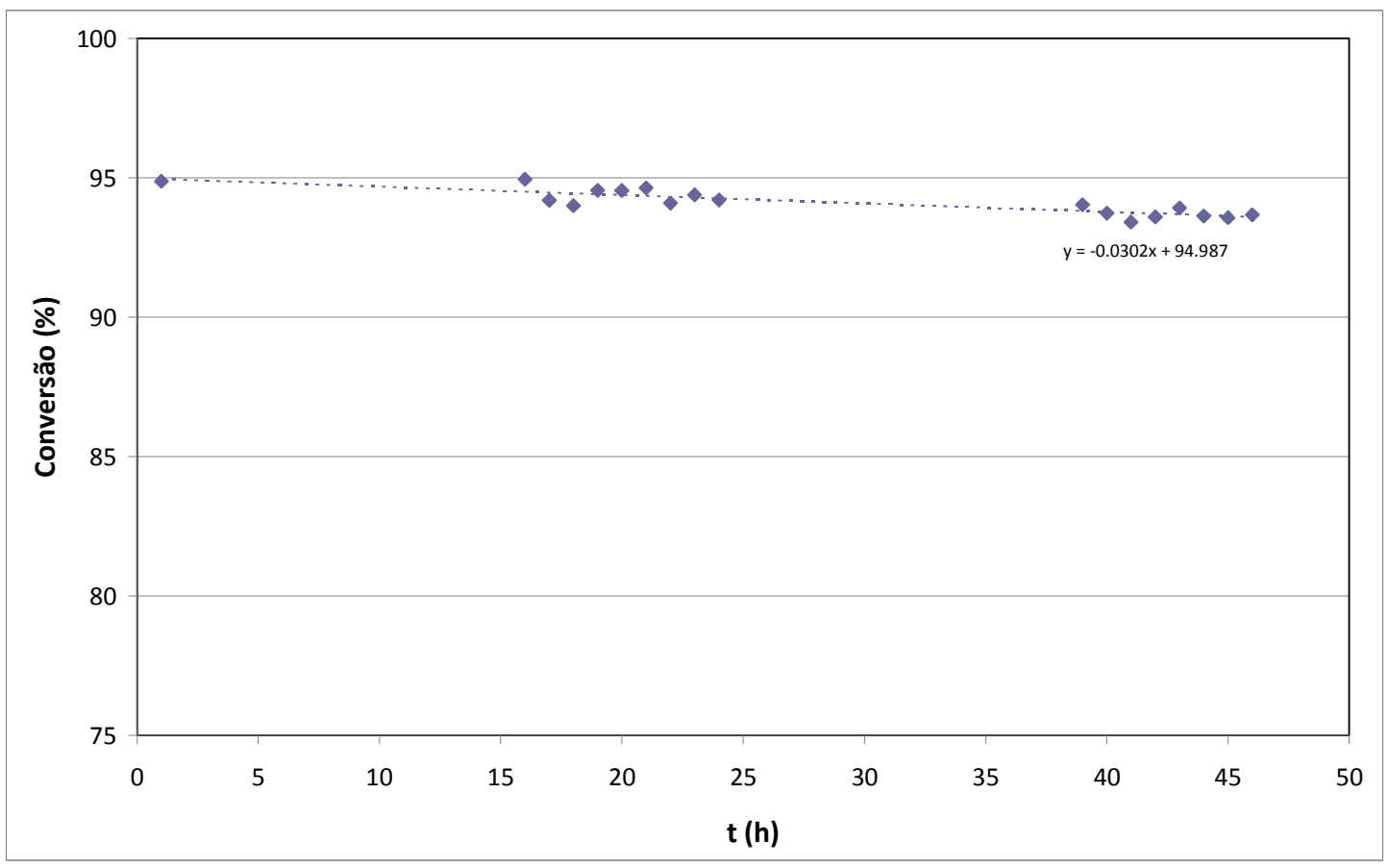

Figura 6- Teste em leito fixo.

Tabela 3 - Eventos térmicos detectados através de termogravimetria dos três açúcares.

\begin{tabular}{l|cc|c}
\multicolumn{1}{c|}{ Açúcar } & \multicolumn{2}{|c|}{ Fusão (pico e faixa) } & Caramelização (faixa) \\
\hline Frutose & $133^{\circ} \mathrm{C}$ & $110^{\circ} \mathrm{C}-160^{\circ} \mathrm{C}$ & $160^{\circ} \mathrm{C}-320^{\circ} \mathrm{C}$ \\
Glicose & $162^{\circ} \mathrm{C}$ & $140^{\circ} \mathrm{C}-200^{\circ} \mathrm{C}$ & $200^{\circ} \mathrm{C}-350^{\circ} \mathrm{C}$ \\
Sacarose & $182^{\circ} \mathrm{C}$ & $140^{\circ} \mathrm{C}-200^{\circ} \mathrm{C}$ & $200^{\circ} \mathrm{C}-350^{\circ} \mathrm{C}$
\end{tabular}




\section{CONCLUSÕES}

A reação de hidrólise da sacarose pode ser conduzida com a resina comercial Amberlyst 36 até conversão próxima a $100 \%$, na condição ótima obtida através do planejamento experimental. A diferença entre as seletividades em regime contínuo e a avaliação térmica dos açúcares indica que nas condições empregadas, apesar do bom desempenho do catalisador para a conversão de uma solução concentrada de sacarose, ocorre degradação da frutose formada durante a reação.

\section{REFERÊNCIAS}

ABBADI, A.; GOTLEIEB, K. F.; BEKKUN, H. V. Study on solid acid catalyzed hydrolysis of maltose related polysaccharides. Starch, v. 50, p. 23-28, 1998.

ALMEIDA, R. M.; JIANRONG, L.; NEDERLOF, C.; O'CONNOR, P.; MAKKEE, M.; MOULIJN, J. A. Cellulose conversion to isosorbide in molten salt hydrate media. ChemSuschem, v. 3, p. 325-328, 2010.

BODAMER, G.; KUNIN, R. Heterogeneous catalytic inversion of sucrose with cation exchange resins. Industrial \&Engineering Chemistry, v. 43, p. 1082-1085, 1951.

BUTTERSACK, C.; LAKETIC, D. hydrolysis of sucrose by dealuminated Y-zeolites. Journal of molecular catalysis, v. 94, p. 283-290, 1994.

CARLINI, C.; GIUTTARI, M.; GALLETTI, A. M. R.; SBRANA, G.; ARMAROLI, T.; BUSCA, G. Selective saccharides dehydration to 5-hydroxymethyl-2furaldehyde by heterogeneous niobium catalysts. Applied Catalysis A: General, v. 183, p. 295-302, 1999.

MARZO, M.; GERVASINI, A.; CARNITI, P. Hydrolysis of disaccharides over solid acids catalysts under green conditions. Carbohydrate research, v. 347, p. 23-31, 2012.

MOREAU, C.; DURAN, R.; ALIES, F.; COTILlON, M.; FRUTZ, T.; THÉOLEYRE, M-A. Hydrolysis of sucrose in the presence of H-form zeolites. Industrial Crops and Products, v. 11, p. 237-242, 2000.

WHYMAN, C. E. Aqueous pretreatment of plant biomass for biological and chemical conversion to fuels and chemicals. Reino Unido: Editora Wiley, 2013.

QUITETE, C. P. B.; XIMENES, V. L.; FRAGA, A. C.; BICUDO, A. A. Hidrólise da celobiose utilizando sólidos ácidos. Anais do $17^{\circ}$ Congresso Brasileiro de Catálise, Gramado, 2013. 